\title{
Papers
}

\section{Risk score for predicting death, myocardial infarction, and stroke in patients with stable angina, based on a large randomised trial cohort of patients}

Tim C Clayton, Jacobus Lubsen, Stuart J Pocock, Zoltán Vokó, Bridget-Anne Kirwan, Keith A A Fox, Philip A

Poole-Wilson, on behalf of the ACTION investigators

\begin{abstract}
Objective To derive a risk score for the combination of death from all causes, myocardial infarction, and disabling stroke in patients with stable symptomatic angina who require treatment for angina and have preserved left ventricular function.

Design Multivariate Cox regression analysis of data from a large multicentre trial.

Setting Outpatient cardiology clinics in western Europe, Israel, Canada, Australia, and New Zealand.

Participants 7311 patients with all required data available.

Main outcome measure Death from any cause or myocardial infarction or disabling stroke during a mean follow-up of 4.9 years.

Results 1063 patients either died from any cause or sustained myocardial infarction or disabling stroke. The five year risk of this composite ranged from $4 \%$ for patients in the lowest tenth of risk to $35 \%$ for patients in the highest tenth. The risk score combines 16 routinely available clinical variables (in order of decreasing contribution): age, left ventricular ejection fraction, smoking, white blood cell count, diabetes, casual blood glucose concentration, creatinine concentration, previous stroke, at least one angina attack a week, coronary angiographic findings (if available), lipid lowering treatment, QT interval, systolic blood pressure $\geq 155 \mathrm{~mm} \mathrm{Hg}$, number of drugs used for angina, previous myocardial infarction, and sex. Fitting the same model separately to all cause death, myocardial infarction, and stroke gave similar results. The risk score did not seem to predict the nature of the event (death in 39\%, myocardial infarction in 46\%, and disabling stroke in 15\%) or the incidence of angiography or revascularisation, which occurred in 29\% of patients. Conclusion This risk score is an objective aid in deciding on further management of patients with stable angina with the aim of reducing serious outcome events. The score can also be used in planning future trials.
\end{abstract}

\section{Introduction}

While patients with stable angina have low mortality, ${ }^{1-3}$ the risk of myocardial infarction and stroke remains substantial and quality of life is often reduced by symptoms. In managing these patients, physicians face complex choices concerning secondary prevention, drugs to treat angina, and indications for coronary angiography and revascularisation.

Because the absolute benefit of any treatment to improve prognosis depends on the risk of disease events, such risks require consideration, especially if treatment has possible complications. Scoring cardiovascular risk was first developed in Framingham. ${ }^{5}$ Many studies have followed, mainly for scoring risk in the primary prevention of cardiovascular diseases. ${ }^{6}$ No similar risk score is available for patients with angina.

Development of a risk score requires a large comprehensive database on patients followed for several years. A potential source is data from large randomised clinical trials. ${ }^{7}$ We used data from the ACTION trial (a coronary disease trial investigating outcome with nifedipine GITS), which followed 7665 patients with stable symptomatic angina for a mean of 4.9 years, ${ }^{3}$ to develop a score for predicting the combined risk of death from any cause, myocardial infarction, and stroke.

\section{Methods}

In the ACTION trial, eligible patients had stable symptomatic angina requiring treatment and either previous myocardial infarction or proved angiographic coronary artery disease. Patients without a previous myocardial infarction or coronary angiography could participate only if there was a positive result on an exercise or perfusion test. ${ }^{38}$ Key exclusions were ejection fraction below $40 \%$, clinically significant heart failure, major cardiovascular event or intervention within the past three months, planned coronary angiography or intervention, and known intolerance to dihydropyridines.

Patients were randomly assigned long acting nifedipine GITS or placebo and were seen regularly at outpatient clinics and contacted by telephone thereafter. The mean follow-up until death or the end of the study was 4.9 years.

Individual baseline data available included demographics, medical history, cardiovascular risk factors, current symptoms of angina and functional status, current treatments, past use of calcium channel blockers, results of non-fasting laboratory tests, left ventricular ejection fraction mostly measured by echocardiography, standard 12 lead electrocardiography findings, routine cuff blood pressure, pulse rate, and the results of previous angiography if available (baseline angiography was not mandated).

We fitted multivariate Cox proportional hazard models for the outcome time to death, myocardial infarction, or disabling stroke as adjudicated by the critical events committee, ${ }^{38}$ using patients who had no missing values for the predictor variables.

Examples of applying the risk score can be found on bmj.com 
We represented binary or categorical variables by indicator variables coded 0 or 1 . Quantitative variables were investigated for non-linearity, and cut off values were determined when appropriate. For instance, for age, 60 years was the cut off value whereby any age $<60$ contributed the same risk. Always entering age, sex, and previous myocardial infarction, we added other variables to the final model using $\mathrm{P}<0.01$ as inclusion criterion. We ignored nifedipine as it had no effect on this combined end point (hazard ratio 1.01, 95\% confidence interval 0.90 to 1.14$)^{3}{ }^{3}$

We expressed each variable's strength of predictive contribution by its z score (the model coefficient divided by its standard error) and quantified each variable's predictive power as a hazard ratio with $95 \%$ confidence interval. To assess the predictive power separately for each of death from any cause, myocardial infarction, and disabling stroke, we refitted the final model using the same covariates for each of the three outcomes concerned.

For each patient, we calculated a risk score by multiplying each coefficient in the final model by 10 , then by the patient's variable value, and then summed up the results. From the distribution of the risk scores, we formed equal sized tenths of increasing risk. Model calibration was evaluated by comparing actual and predicted outcomes in each tenth of risk, the actual outcome being the five year Kaplan-Meier probability estimate and the expected probability being calculated from the final model. The internal validity of the final model was assessed by the bootstrap resampling technique. ${ }^{9}$ For each of 100 bootstrap samples the model was refitted and tested on the original sample to obtain an estimate of predictive accuracy corrected for bias. This revealed no overoptimism in the final model's predictive discrimination. We calculated the rate of coronary intervention including coronary angiography, percutaneous coronary intervention, or bypass surgery in the tenths of the risk score to study the relation between the predicted risk of major cardiovascular events and the use of coronary interventions.

\section{Results}

Table 1 shows the 16 variables, with their $\mathrm{z}$ scores and Cox regression coefficients, that were in the final model as derived for 7311 patients $(95 \%)$ with complete information. In all 1063 patients died or sustained myocardial infarction or disabling stroke. Table 1 also shows how we derived the risk score (see bmj.com for examples). Table 2 presents the corresponding hazard ratios for the composite of death, myocardial infarction, and stroke. Age was the strongest predictor (hazard ratio 1.73 for each decade of age $>60$ ). Male sex (hazard ratio 1.17) was of borderline significance $(\mathrm{P}=0.06)$ but was retained for completeness. Diabetes and stroke were the strongest predictors from clinical history. Patients with known three or more vessel disease had raised risk. The final model also includes several standard risk factors, such as current smoking, and the laboratory results for white blood cell count, creatinine, and glucose. The latter contributed most to prediction in those without diabetes and was not predictive in those with diabetes treated with insulin. Other predictors included were left ventricular ejection fraction, a prolonged QT interval, use of lipid lowering drugs, and the number of drugs used for angina (including past use of calcium blockers).

Table 2 also presents hazard ratios for the individual events of death, myocardial infarction, and disabling stroke with the same variables as for the combined end point. Patterns of risk factors were broadly similar, though risk of stroke was more strongly linked to raised blood pressure but unrelated to white cell count, angiographic data, previous myocardial infarction, and sex.
Figure 1 shows the distribution of the risk score (multiplied by ten) based on the coefficients and definitions given in table 1 , and also its association with the probability of death, myocardial infarction, and disabling stroke within five years. Figure 2 shows good agreement between the observed and expected proportions of patients having this composite event within five years. Among patients with risk scores in the top and bottom tenths, the probability of an event was $35 \%$ and $4 \%$, respectively. The Kaplan-Meier plots for each tenth showed that event rates were essentially constant over six years' follow-up.

Table 3 shows the number of patients who sustained the composite of death, myocardial infarction, and disabling stroke by tenth of risk with the corresponding event rates. First events comprised $417(39 \%)$ deaths, $490(46 \%)$ myocardial infarctions, and $156(15 \%)$ disabling strokes, and the distribution of first event did not vary substantially by tenth of risk. Table 3 also shows the distribution by tenth of risk of the 2147 patients (29\%) who had a coronary angiogram or coronary revascularisation procedure during follow-up. There was no relation between these intervention rates and the risk of death, myocardial infarction, and stroke.

\section{Discussion}

Our study confirms that most patients with stable symptomatic coronary disease have a relatively good prognosis. ${ }^{12}$ Our main finding is the large variation in risk of death, myocardial infarction, and disabling stroke between patients that can be determined from an easily calculated risk score using standard clinical information. Others have previously studied predictors of clinical outcome in patients with stable angina ${ }^{10}$ but on fewer patients followed for a shorter period.

That patients in the top $10 \%$ of risk had ten times the risk of patients in the bottom $10 \%$ of risk is clinically relevant. Coronary revascularisation prolongs survival only in high risk patients. ${ }^{4}$ Hence, risk stratification using our score helps to identify patients with stable angina for whom elective revascularisation might improve prognosis. Risk stratification aids decisions on secondary preventive medical management, especially when limited resources exist for coronary angiography and revascularisation. Patients at high risk of serious clinical events can be given priority so as to avoid such events while they are waiting for an invasive procedure.

We found no relation between the use of procedures related to treatment of angina and risk of death, myocardial infarction, and stroke, which suggests that events related to plaque rupture (cardiovascular death, myocardial infarction) have a different pathology and different risk factors from worsening angina caused by progression of coronary artery disease. This implies that our risk score contains no information regarding whether a patient will need a coronary angiogram or revascularisation procedure because of worsening angina. Hence, risk stratification has no bearing on prescribing drug treatment for angina to avoid the need for coronary revascularisation.

We deliberately focused prediction on important life events: death, unequivocal myocardial infarction, and stroke that is disabling. Contrary to current recommendations, ${ }^{11}$ our definition of myocardial infarction did not include patients with chest pain and raised troponin concentrations. The definition of stroke excluded events without lasting disability. ${ }^{3}$ We included 206 noncardiovascular deaths ( $19 \%$ of all deaths), which may reduce the predictive power of risk factors particularly related to cardiovascular outcomes, but none the less makes the model more useful for clinical application. 
Table 1 Predictors of death, myocardial infarction (MI), or disabling stroke for 7311 participants in the ACTION trial (Cox proportional hazards analysis). Figures are numbers (percentages) unless stated otherwise

\begin{tabular}{|c|c|c|c|c|c|}
\hline & $\begin{array}{l}\text { Death, MI or stroke } \\
(n=1063)^{*}\end{array}$ & $\begin{array}{l}\text { No death, MI or stroke } \\
\qquad(n=6248)^{\star}\end{array}$ & z score & Coefficient & Contribution to risk score \\
\hline Mean (SD) age (years) & $66.5(9.5)$ & $63.0(9.2)$ & 10.77 & 0.55 & 0 when age $\leq 60$ years or add per 10 years $>60$ years \\
\hline Mean (SD) ejection fraction (\%) & $46.7(6.6)$ & $48.6(6.3)$ & 6.47 & 0.17 & 0 when $\geq 60 \%$ or add per $5 \%<60 \%$ \\
\hline \multicolumn{6}{|l|}{ Smokingł: } \\
\hline Never & $260(24)$ & $1784(29)$ & - & - & 0 \\
\hline Ex-smoker & $560(53)$ & $3417(55)$ & 1.54 & 0.12 & Add if applicable \\
\hline Current & $243(23)$ & $1047(17)$ & 6.12 & 0.60 & Add if applicable \\
\hline Mean (SD) white blood cells $\left(10^{9} / \mathrm{l}\right)$ & $7.4(2.5)$ & $7.0(1.8)$ & 6.07 & 0.068 & 0 when $\leq 510^{9} / l$ or add per $10^{9} / l>5$ \\
\hline \multicolumn{6}{|l|}{ Diabetesł: } \\
\hline No diabetes & $848(80)$ & $5393(86)$ & - & - & 0 \\
\hline Non-ID diabetes & $167(16)$ & $727(12)$ & 1.06 & 0.13 & Add if applicable \\
\hline ID diabetes & $48(5)$ & $128(2)$ & 5.61 & 0.85 & Add if applicable \\
\hline Mean (SD) glucose, no diabetes $(\mathrm{mg} / \mathrm{dl}) \S$ & $103(26)$ & $99(20)$ & 4.68 & 0.072 & 0 when $\leq 100 \mathrm{mg} / \mathrm{dl}$ or add per $10 \mathrm{mg} / \mathrm{dl}>100 \mathrm{mg} / \mathrm{dl}$ \\
\hline Mean (SD) glucose, non-ID diabetes (mg/dl)§ & $189(79)$ & $168(65)$ & 3.36 & 0.032 & 0 when $\leq 100 \mathrm{mg} / \mathrm{dl}$ or add per $10 \mathrm{mg} / \mathrm{dl}>100 \mathrm{mg} / \mathrm{dl}$ \\
\hline Mean (SD) creatinine $(\mathrm{mg} / \mathrm{dl})$ 川 & $1.14(0.25)$ & $1.08(0.21)$ & 4.27 & 0.078 & 0 when $\leq 1.15 \mathrm{mg} / \mathrm{dl}$ or add per $0.1 \mathrm{mg} / \mathrm{dl}>1.15 \mathrm{mg} / \mathrm{dl}$ \\
\hline Previous stroke $e^{\star *}$ & $50(5)$ & $116(2)$ & 3.59 & 0.53 & Add if yes \\
\hline Angina attack $\geq 1 /$ week $^{\star \star}$ & $364(34)$ & $1750(28)$ & 3.42 & 0.22 & Add if applicable \\
\hline \multicolumn{6}{|l|}{ Previous angiographył: } \\
\hline Never done & $350(33)$ & $1842(29)$ & 1.50 & 0.11 & Add if applicable \\
\hline $0-2$ vessel disease†† & $421(40)$ & 3069 (49) & - & - & Add 0 if applicable \\
\hline$\geq 3$ vessel disease & $292(27)$ & $1337(21)$ & 3.23 & 0.25 & Add if applicable \\
\hline No lipid lowering therapy & $406(38)$ & $1950(31)$ & 3.20 & 0.21 & Add if not on therapy \\
\hline QT interval (12 lead ECG) $\geq 430 \mathrm{msec}^{\text {** }}$ & $238(22)$ & $1096(18)$ & 3.05 & 0.23 & Add if applicable \\
\hline Systolic blood pressure $\geq 155 \mathrm{~mm} \mathrm{Hg}$ * & $275(26)$ & $1097(18)$ & 2.84 & 0.21 & Add if applicable \\
\hline \multicolumn{6}{|l|}{ No of drugs for angina: } \\
\hline 0 & $8(1)$ & $53(1)$ & - & - & \\
\hline 1 & $268(25)$ & $1953(31)$ & 2.76 & 0.13 & Add once for each drug used \\
\hline 2 & $626(59)$ & $3487(56)$ & & & \\
\hline 3 & $161(15)$ & 755 (12) & & & \\
\hline Previous $\mathrm{MI}^{* *}$ & $597(56)$ & $3118(50)$ & 2.16 & 0.14 & Add if yes \\
\hline$\overline{\text { Male }^{\star \star}}$ & $863(81)$ & 4944 (79) & 1.87 & 0.16 & Add if male \\
\hline
\end{tabular}

ID=insulin dependent.

* Model is based on 7311 patients with values for all variables in model, of whom 1063 had the combined event of death, MI, or disabling stroke.

tCoefficient divided by its $S E$. Larger values indicate more highly significant risk factor: $z$ scores of $1.96,2.58,3.29$, and 3.89 correspond to $P=0.05, P=0.01, P=0.001$, and $P=0.0001$, respectively.

†Categorical variable represented by two indicator variables in model, reference category shown.

$\S \times 0.0555$ to convert values to $\mathrm{mmol} / \mathrm{l}$.

q $\times 88.4$ to convert values to $\mu \mathrm{mol} / /$.

**Binary variable, reference category not shown.

$\dagger+$ No of major coronary arteries with clinically significant lesions for all coronary angiograms performed. Left main disease was counted as two vessel disease.

Many of the risk factors we identified are not surprising. Age, ejection fraction, smoking, and diabetes are all known predictors from other studies. Statins have been shown to reduce mortality, myocardial infarction, and stroke in several settings. ${ }^{12}$ The link between white blood cell count and coronary disease risk is established. ${ }^{13}$ Other markers of inflammation such as $\mathrm{C}$ reactive protein were not measured. Raised serum creatinine concentration is linked to adverse cardiovascular outcome in several populations, including people with hypertension, ${ }^{14}$ myocardial infarction, ${ }^{15}$ and acute coronary syndrome. ${ }^{16}$ The extent of angiographic coronary disease is a well established risk factor. ${ }^{4}$ That patients who never had coronary angiography had a slightly higher risk than patients who had known zero to two vessel disease seems surprising: one likely explanation is that the former includes some patients with extensive, albeit undocumented, coronary disease. One could confine the analysis to patients with angiographic information, but this would limit the clinical relevance as many patients with angina never have coronary angiography.

The possibility exists that design issues in the ACTION trial could influence our prognostic models. For instance, patients on a calcium channel blocker could not participate in the trial, though $22 \%$ of patients had used a calcium channel blocker in the past. Past use of a calcium channel blocker was a significant predictor in the present analysis and we have retained such past use in the model by incorporating it in the number of drugs used for angina. The present risk score is limited to patients with preserved left ventricular function who did not have any condition, other than coronary artery disease, that limits life expectancy.

Our risk score may also be useful in planning future clinical trials in similar patients by, for instance, enabling them to exclude low risk patients.

The contribution of investigators, committee members and other ACTION study personnel as listed elsewhere ${ }^{3}$; the support of Bayer Healthcare AG; and the contributions of all participating patients over the course of the prolonged follow-up required are gratefully acknowledged. To make the risk score user friendly a website www.anginarisk.org is under development for ease of implementation.

Contributors: TCC carried out the statistical analysis. JL, SJP, B-AK, and ZV wrote the paper. All authors were involved in the design of the analysis, interpretation of the results, and improvements to the manuscript. All authors except ZV were also involved in the ACTION trial. SJP is the guarantor.

Funding: The ACTION trial was supported by Bayer Healthcare AG, Wuppertal, Germany.

Competing interests: The ACTION study was carried out by an independent steering committee and research group. JL, KAAF and PAP-W have served as consultants to, or received travel expenses, payment for speaking at meetings, or funding for research from, other pharmaceutical companies. 
Table 2 Predictors of death, myocardial infarction (MI), and disabling stroke for 7311 participants in the ACTION trial (Cox proportional hazards analysis). Figures are hazard ratios (95\% confidence intervals)

\begin{tabular}{|c|c|c|c|c|}
\hline & Death, MI, or stroke $(n=1063)$ & Death $(n=569)$ & MI $(n=495)$ & Stroke $(n=170)$ \\
\hline Age per 10 years $>60$ & 1.73 (1.57 to 1.92$)$ & 2.30 (2.01 to 2.64$)$ & 1.45 (1.25 to 1.69$)$ & 1.75 (1.37 to 2.24$)$ \\
\hline Ejection fraction per $5 \%<60$ & 1.19 (1.13 to 1.25$)$ & 1.26 (1.17 to 1.35$)$ & 1.14 (1.06 to 1.23$)$ & $1.24(1.09$ to 1.41$)$ \\
\hline \multicolumn{5}{|l|}{ Smoking*: } \\
\hline Never & 1.00 & 1.00 & 1.00 & 1.00 \\
\hline Ex-smoker & 1.13 (0.97 to 1.32$)$ & 1.19 (0.96 to 1.48$)$ & 0.99 (0.79 to 1.24$)$ & 1.42 (0.95 to 2.13$)$ \\
\hline Current & 1.82 (1.50 to 2.20$)$ & 2.20 (1.69 to 2.85$)$ & 1.39 (1.05 to 1.84$)$ & 2.44 (1.49 to 3.99$)$ \\
\hline White blood cells per $10^{9} / l>5$ & 1.07 (1.05 to 1.09$)$ & 1.09 (1.07 to 1.12$)$ & 1.05 (1.01 to 1.10$)$ & $1.00(0.92$ to 1.09$)$ \\
\hline \multicolumn{5}{|l|}{ Diabetes*: } \\
\hline No diabetes & 1.00 & 1.00 & 1.00 & 1.00 \\
\hline Non-ID diabetes & 1.14 (0.90 to 1.44$)$ & 0.93 (0.66 to 1.32$)$ & $1.14(0.81$ to 1.60$)$ & 1.75 (1.06 to 2.90$)$ \\
\hline ID diabetes & 2.33 (1.74 to 3.14$)$ & 3.44 (2.40 to 4.94$)$ & 2.62 (1.75 to 3.93$)$ & $0.56(0.14$ to 2.29$)$ \\
\hline Glucose per 10 mg/dl >100† (no diabetes) & 1.08 (1.04 to 1.11$)$ & $1.10(1.06$ to 1.14$)$ & $1.05(1.00$ to 1.10$)$ & $1.07(0.98$ to 1.15$)$ \\
\hline Glucose per $10 \mathrm{mg} / \mathrm{dl}>100 \dagger$ (non-ID diabetes) & 1.03 (1.01 to 1.05$)$ & 1.04 (1.01 to 1.07$)$ & $1.03(1.00$ to 1.06$)$ & 1.03 (0.99 to 1.07$)$ \\
\hline Creatinine per $0.1 \mathrm{mg} / \mathrm{dl}>1.15 \ddagger$ & 1.08 (1.04 to 1.12$)$ & 1.09 (1.04 to 1.14$)$ & $1.08(1.02$ to 1.14$)$ & $1.06(0.97$ to 1.16$)$ \\
\hline Previous stroke§ & 1.70 (1.27 to 2.28$)$ & 1.74 (1.19 to 2.54$)$ & 1.50 (0.95 to 2.36$)$ & 4.28 (2.60 to 7.06$)$ \\
\hline Angina attack $\geq 1 /$ week§ & 1.25 (1.10 to 1.42$)$ & 1.27 (1.07 to 1.51$)$ & 1.21 (1.00 to 1.46$)$ & 1.16 (0.84 to 1.61$)$ \\
\hline \multicolumn{5}{|l|}{ Previous angiography*: } \\
\hline Never done & 1.12 (0.97 to 1.30$)$ & $1.16(0.95$ to 1.41$)$ & $1.20(0.96$ to 1.49$)$ & 1.10 (0.77 to 1.58$)$ \\
\hline 0-2 vessel diseasen & 1.00 & 1.00 & 1.00 & 1.00 \\
\hline$\geq 3$ vessel disease & 1.28 (1.10 to 1.50$)$ & $1.14(0.92$ to 1.41$)$ & $1.50(1.21$ to 1.87$)$ & $1.06(0.72$ to 1.57$)$ \\
\hline No lipid lowering therapy§ & 1.23 (1.08 to 1.40$)$ & $1.33(1.12$ to 1.58$)$ & 1.10 (0.91 to 1.33$)$ & $1.09(0.79$ to 1.51$)$ \\
\hline QT interval (12-lead ECG) $\geq 430 \mathrm{msec} \S$ & 1.26 (1.08 to 1.45$)$ & 1.52 (1.26 to 1.84$)$ & 1.08 (0.87 to 1.35$)$ & $1.42(1.00$ to 2.01$)$ \\
\hline Systolic blood pressure $\geq 155 \mathrm{~mm} \mathrm{Hg \S}$ & 1.23 (1.07 to 1.42$)$ & 1.18 (0.98 to 1.43$)$ & 1.09 (0.88 to 1.35$)$ & 1.69 (1.22 to 2.36$)$ \\
\hline For each additional drug for angina & 1.14 (1.04 to 1.25$)$ & 1.09 (0.96 to 1.24) & 1.20 (1.05 to 1.38$)$ & $1.21(0.96$ to 1.54$)$ \\
\hline Previous MI§ & 1.15 (1.01 to 1.30$)$ & $1.10(0.92$ to 1.30$)$ & $1.16(0.96$ to 1.39$)$ & $1.01(0.74$ to 1.38$)$ \\
\hline Male§ & 1.17 (0.99 to 1.39$)$ & 1.21 (0.96 to 1.52$)$ & 1.24 (0.97 to 1.59$)$ & 0.88 (0.59 to 1.30$)$ \\
\hline
\end{tabular}

$\mathrm{ID}=$ insulin dependent.

${ }^{*}$ Categorical variable represented by two indicator variables in model, reference category shown.

$+\times 0.0555$ to convert values to $\mathrm{mmol} / \mathrm{l}$.

$\neq \times 88.4$ to convert values to $\mu \mathrm{mol} / \mathrm{l}$.

$\S$ Binary variable, reference category not shown

१No of major coronary arteries with clinically significant lesions for all coronary angiograms performed. Left main disease was counted as two vessel disease.

BAK and JL are full time employees of SOCAR Research SA, which managed the study.

Ethical approval: Not required.

1 Yusuf S, Sleight P, Pogue J, Bosch J, Davies R, Dagenais G. Effects of an angiotensin-converting-enzyme inhibitor, ramipril, on cardiovascular events in high-risk patients. The heart outcomes prevention evaluation study investigators. $N$ high-risk patients. The hear
Engl J Med 2000;342:145-53.

2 Fox KM. Efficacy of perindopril in reduction of cardiovascular events among patients with stable coronary artery disease: randomised, double-blind, placebo-controlled, multicentre trial (the EUROPA study). Lancet 2003;362:782-8.

3 Poole-Wilson PA, Lubsen J, Kirwan BA, van Dalen FJ, Wagener G, Danchin N, et al. Effect of long-acting nifedipine on mortality and cardiovascular morbidity in patient with stable angina requiring treatment (ACTION trial): randomised controlled trial. Lancet 2004;364:849-57.

4 O'Toole L, Grech ED. Chronic stable angina: treatment options. BMJ 2003;326:1185-8.

5 Anderson KM, Odell PM, Wilson PW, Kannel WB. Cardiovascular disease risk profiles. Am Heart J 1991;121:293-8.

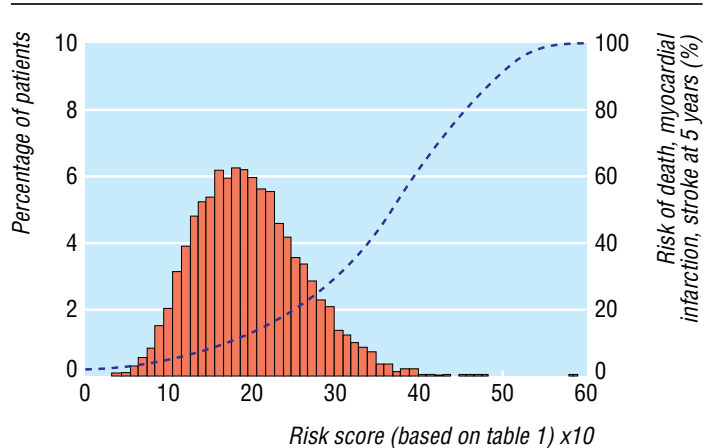

Fig 1 Distribution of risk score for 7311 patients with stable angina and model-based relation between risk score and probability of dying or sustaining myocardial infarction or disabling stroke in five years
6 De Backer G, Ambrosioni E, Borch-Johnsen K, Brotons C, Cifkova R, Dallongeville J, et al. European guidelines on cardiovascular disease prevention in clinical practice. Thirc joint task force of European and other societies on cardiovascular disease prevention joint task force of European and other societies

7 Pocock SJ, McCormack V, Gueyffier F, Boutitie F, Fagard RH, Boissel JP. A score for predicting risk of death from cardiovascular disease in adults with raised blood pressure, based on individual patient data from randomised controlled trials. $B M$ J 2001;323:75-81.

8 Lubsen J, Poole-Wilson PA, Pocock SJ, van Dalen FJ, Baumann J, Kirwan BA, et al. Design and current status of ACTION: a coronary disease trial investigating outcome with nifedipine GITS. Gastro-intestinal therapeutic system. Eur Heart J 1998;19(suppl I):20-32.

9 Harrell FE Jr, Lee KL, Califf RM, Pryor DB, Rosati RA. Regression modelling strategies for improved prognostic prediction. Stat Med 1984;3:143-52.

10 Daly C, Norrie J. Murdoch DL, Ford I, Dargie HJ, Fox K. The value of routine non-invasive tests to predict clinical outcome in stable angina. Eur Heart $J$ 2003;24:532-40

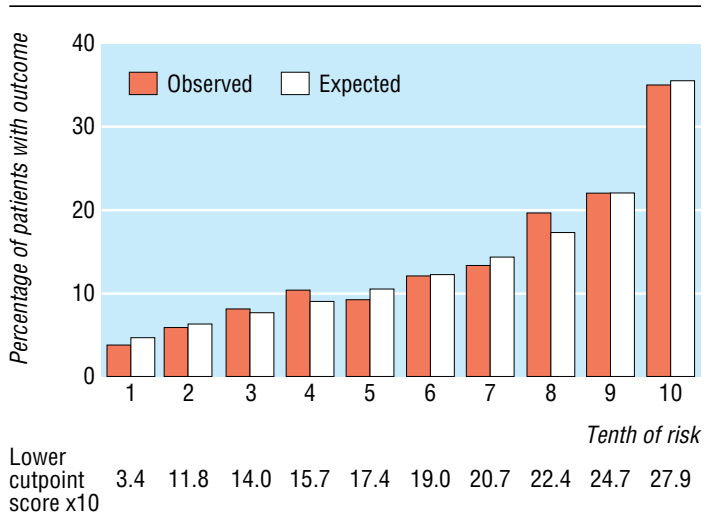

Fig 2 Expected and observed probabilities of dying or sustaining myocardial infarction or disabling stroke in five years by tenth of risk score for patients with stable angina 
Table 3 Occurrence of death, myocardial infarction (MI), or disabling stroke and rate of coronary interventions by tenth of risk

\begin{tabular}{|c|c|c|c|c|c|}
\hline \multirow{2}{*}{$\begin{array}{l}\text { Tenth of risk for death, MI, } \\
\text { or stroke* }\end{array}$} & \multirow{2}{*}{$\begin{array}{l}\text { No of deaths, Mls, or strokes } \\
\text { (No/100 person years) }\end{array}$} & \multicolumn{3}{|c|}{ First event (\% of total death, MI, or stroke) } & \multirow[b]{2}{*}{ Any interventiont (№/100 person years) } \\
\hline & & Death $(\%)$ & MI (\%) & Stroke (\%) & \\
\hline$\overline{1 s t}$ & $31(0.86)$ & $5(16)$ & $21(67)$ & $5(16)$ & 188 (5.92) \\
\hline 2nd & $43(1.18)$ & $9(21)$ & $25(58)$ & $9(21)$ & $210(6.74)$ \\
\hline $3 \mathrm{rd}$ & $64(1.79)$ & $23(36)$ & $37(58)$ & $4(6)$ & $238(8.04)$ \\
\hline 4th & $77(2.18)$ & $28(36)$ & $41(53)$ & $8(10)$ & $241(8.22)$ \\
\hline$\overline{5 \text { th }}$ & $72(1.98)$ & $30(42)$ & $32(44)$ & $10(14)$ & $208(6.63)$ \\
\hline 6th & $90(2.56)$ & $29(32)$ & $49(54)$ & $12(13)$ & $227(7.70)$ \\
\hline 7 th & $101(2.86)$ & $42(42)$ & $44(44)$ & $15(15)$ & $205(6.66)$ \\
\hline 8th & $143(4.20)$ & $63(44)$ & $61(43)$ & $19(13)$ & $211(7.08)$ \\
\hline 9th & $169(5.04)$ & $65(38)$ & $70(41)$ & $34(20)$ & $214(7.27)$ \\
\hline 10th & $273(8.93)$ & $123(45)$ & $110(40)$ & $40(15)$ & $205(7.32)$ \\
\hline Total $(n=7311)$ & $1063(3.05)$ & 417 (39) & $490(46)$ & $156(15)$ & $2147(7.14)$ \\
\hline
\end{tabular}

*Tenths 1-9 consist of 731 patients each, last 10th consists of 732 patients.

†Coronary angiography, percutaneous coronary intervention, or bypass surgery.

11 Alpert JS, Thygesen K, Antman E, Bassand JP. Myocardial infarction redefined-a consensus document of the Joint European Society of Cardiology/American College of Cardiology committee for the redefinition of myocardial infarction. J Am Coll Cardiol 2000;36:959-69.

12 Wei L, Ebrahim S, Bartlett C, Davey PG, Sullivan FM, Macdonald TM. Statin use in the secondary prevention of coronary heart disease in primary care: cohort study and comparison of inclusion and outcome with patients in randomised trials. BMJ comparison of in

\section{What is already known on this topic}

Many factors are known to affect the risk of death, myocardial infarction, or stroke in patients with stable symptomatic coronary disease (angina pectoris)

There is no method available to assess a patient's overall risk based on routine clinical information

\section{What this study adds}

A new score uses 16 clinical variables to quantify the combined risk of death from any cause, myocardial infarction, and disabling stroke

Patients with risk scores in the highest tenth have 10 times the risk of patients with scores in the lowest tenth

The use of invasive procedures such as coronary angiography or revascularisation undertaken to relieve symptoms of angina was not related to the composite risk
13 Pearson TA, Mensah GA, Hong Y, Smith SC Jr. CDC/AHA workshop on markers of inflammation and cardiovascular disease: application to clinical and public health practice: overview. Circulation 2004;110:e543-4.

14 Schillaci G, Reboldi G, Verdecchia P. High-normal serum creatinine concentration is a predictor of cardiovascular risk in essential hypertension. Arch Intern Med 2001;161:886-91.

15 Matts JP, Karnegis JN, Campos CT, Fitch LL, Johnson JW, Buchwald H. Serum creatinine as an independent predictor of coronary heart disease mortality in normotensive survivors of myocardial infarction. POSCH Group.J Fam Pract 1993;36:497-503.

16 Eagle KA, Lim MJ, Dabbous OH, Pieper KS, Goldberg RJ, Van de Werf F, et al. A validated prediction model for all forms of acute coronary syndrome: estimating the risk of 6-month postdischarge death in an international registry. JAMA 2004:291:2727-33.

(Accepted 10 August 2005)

doi 10.1136/bmj.38603.656076.63

Department of Epidemiology and Population Health, London School of Hygiene and Tropical Medicine, London WC1E 7HT

Tim C Clayton lecturer

Stuart J Pocock professor

Department of Epidemiology and Biostatistics, Erasmus Medical Centre, 3000 DR Rotterdam, Netherlands

Jacobus Lubsen professor

SOCAR Research SA, PO Box 2564, CH-1260 Nyon 2, Switzerland

Bridget-Anne Kirwan director

School of Public Health, University of Debrecen, H-4028 Debrecen, Hungary Zoltán Vokó assistant professor

Cardiovascular Research Unit, Edinburgh University, Edinburgh EH16 4SB Keith A A Fox professor

Cardiovascular Sciences, National Heart and Lung Institute, Imperial College London, London SW3 6LY

Philip A Poole-Wilson professor

Correspondence to:J Lubsen jlubsen@compuserve.com 